\title{
Condição de saúde bucal de crianças com deficiência auditiva matriculadas em uma escola de educação especial
}

\section{Oral health status of children with hearing impairment, enrolled in a special education school}

\author{
Bruna Aldrighi Ruas \\ Cilene Gonçalves Borges* \\ Vanessa Polina Pereira Costa** \\ Lisandrea Rocha Schardosim ${ }^{* * *}$
}

\section{Resumo}

Objetivo: avaliar a condição de saúde bucal de escolares entre 6 e 19 anos de idade com deficiência auditiva de uma escola de educação especial. Sujeitos e método: este estudo transversal prospectivo coletou dados sobre condições socioeconômicas, grau de deficiência auditiva e hábitos de higiene bucal por intermédio de um questionário direcionado aos cuidadores. A condição de saúde bucal foi obtida por meio de exame clínico, avaliando o índice de placa visível, o índice de sangramento gengival e o índice de dentes cariados, perdidos e obturados (ceo-d e CPO-D). A análise descritiva foi realizada com valores absolutos e relativos, usando o programa Stata versão 10.0. A associação entre as variáveis independentes e o desfecho do estudo foi verificada por meio dos testes Qui-Quadrado, Exato de Fisher e Mann-Whitney, com nível de significância de 5\%. Resultados: foram avaliados cinquenta escolares, a maioria do sexo masculino, de baixa renda cujo cuidador era do lar. Em relação à saúde bucal, $90 \%$ dos escolares apresentaram placa visível, 22\% tiveram sangramento gengival na sondagem e a média do índice CPO-D foi 1,8. Conclusões: a condição de higiene bucal dos escolares com deficiência auditiva avaliados foi insatisfatória e a doença cárie esteve presente em uma parcela considerável, porém, semelhante à população sem deficiência.

Palavras-chave: Cárie dentária. Estudantes. Pessoas com deficiência auditiva. Saúde bucal.

\section{Introdução}

De acordo com a Organização Mundial de Saúde $(\mathrm{OMS})^{1}$, cerca de $10 \%$ da população mundial é constituída de pessoas com deficiência $(\mathrm{PcD})$, sendo que $15 \%$ são pessoas com deficiência auditiva ${ }^{2}$.

A deficiência auditiva é caracterizada pela incapacidade parcial ou total de se compreender a fala pela audição, variando em diferentes níveis e graus, não se restringindo às funções otológicas, podendo afetar o desenvolvimento da linguagem oral, da aprendizagem bem como da personalidade e de suas relações sociais ${ }^{3}$.

Poucos são os estudos sobre condição de saúde bucal entre as pessoas com deficiência e, apesar de controversos, a maioria dos trabalhos disponíveis demonstra que a prevalência de cárie é alta nesse grupo $^{4-11}$. Dentre os pacientes com deficiência auditiva, essa prevalência também é alta, variando de $46 \%$ a $83,9 \%$, 7,9 .

Informações epidemiológicas sobre doenças bucais, como cárie e periodontopatias, são importantes para a promoção de saúde bucal entre os PcDs e são elementos fundamentais para o desenvolvimento de programas educativos e preventivos ${ }^{12}$. Os poucos estudos disponíveis apresentam variação metodológica e diferenças entre populações e faixa etária, o que dificulta traçar um perfil da condição bucal dessa população.

\footnotetext{
Cirurgiãs-dentistas, Faculdade de Odontologia, Universidade Federal de Pelotas, Pelotas, Brasil.

Doutora em Odontopediatria, pós-doutoranda em Odontopediatria, Programa de Pós-Graduação em Odontologia, Universidade Federal de Pelotas, Pelotas, Brasil.

** Doutora em Estomatologia Clínica. Professora adjunta da Unidade de Clínica Infantil, Departamento de Odontologia Social e Preventiva, Faculdade de Odontologia, Universidade Federal de Pelotas, Pelotas, Brasil.
} 
Assim, este estudo objetiva avaliar a condição de saúde bucal de escolares entre 6 e 19 anos de idade com deficiência auditiva de uma escola de educação especial de referência no município de Pelotas, Rio Grande do Sul, Brasil.

\section{Sujeitos e método}

Este é um estudo transversal prospectivo com uma amostra composta por 50 escolares, com idades entre 6 e 19 anos, com deficiência auditiva, matriculados na Escola Especial Professor Alfredo Dub, em Pelotas, no Rio Grande do Sul, Brasil, assim como seus respectivos pais e/ou cuidadores. Dos 61 escolares matriculados na educação infantil e no ensino fundamental, $57(93,4 \%)$ foram selecionados para o estudo de acordo com os critérios de inclusão: ter entre 6 e 19 anos, estar regularmente matriculado, ter deficiência auditiva (total ou parcial).

O estudo foi aprovado pelo Comitê de Ética em Pesquisa da Faculdade de Odontologia da Universidade Federal de Pelotas (FO-UFPEL), sob Parecer no 01/2014, e autorizado pela direção da escola especial. Os pais e/ou cuidadores foram esclarecidos sobre os objetivos da pesquisa por meio de uma reunião inicial realizada na escola, e o escolar foi incluído após assinatura do termo de consentimento livre e esclarecido e do termo de assentimento livre e esclarecido.

Inicialmente, foi aplicado um questionário aos cuidadores, a fim de coletar dados socioeconômicos, demográficos (gênero, idade, renda familiar, escolaridade e ocupação do cuidador) e sobre os hábitos de higiene bucal do escolar (quem realiza a escovação, com que frequência e como é realizada). A idade foi estratificada em 6 a 12 anos e de 13 a 19 anos para facilitar a comparação com outros estudos que envolvem pacientes com deficiência auditiva.

Para o exame bucal, as avaliadoras passaram por um treinamento teórico e prático sobre os códigos e critérios que foram utilizados em relação ao índice de placa visível (IPV) ${ }^{13}$, índice de sangramento gengival na sondagem (ISG) ${ }^{13}$ e índice de dentes cariados, perdidos e obturados na dentição decídua (ceo-d) e permanente (CPO-D), preconizado pela $\mathrm{OMS}^{1}$. Para avaliar a concordância interexaminador, foi realizada calibração com pacientes da Clínica Infantil da Faculdade de Odontologia da Universidade Federal de Pelotas, obtendo-se um valor de Kappa de 0,91 para cárie e 0,7 para sangramento, sendo ambos os valores considerados satisfatórios para uma das examinadoras, que realizou todos os exames. O exame de placa visível foi incluído no exercício de calibração, mas não na análise de concordância, devido à dificuldade de manter a condição inalterada durante a série de exames. A experiência de cárie foi considerada quando o ceo-d/ CPO-D foi maior que zero.
Todos os exames foram realizados pela mesma examinadora e anotadora. A avaliação do IPV e do ISG foi dicotômica (presente/ausente) e realizada nos dentes índices 16, 11, 26, 36, 31 e 46, empregados no índice de higiene oral simplificado de Greene e Vermillion ${ }^{14}$ (1964). Na ausência de algum dente índice, foi analisado o seu correspondente decíduo ou o dente mais próximo (mesial) da hemiarcada. $\mathrm{O}$ exame foi realizado na própria escola, para tanto, os estudantes sentaram-se em cadeiras escolares e foram utilizadas luz natural, espelho clínico e sonda periodontal comunitária (sonda CPI).

Para a placa dental, utilizou-se o critério de placa visível a olho nu, sem o auxílio de sonda. Foi atribuído o escore 0 para ausência de placa visível e o escore 1 para presença de placa visível. $O$ sangramento foi avaliado contornando-se a margem gengival dos dentes com sonda CPI, de mesial para distal e de vestibular para lingual, suavemente, com aproximadamente $0,5 \mathrm{~mm}$ de profundidade. A ausência de sangramento gengival (escore 0) ou a presença (escore 1) foram observadas após 30 segundos da sondagem.

Ao término da pesquisa, foi entregue ao aluno um kit de saúde bucal, contendo escova de dentes e dentifrício fluoretado, também foi realizada uma atividade educativo-preventiva com os escolares, baseada em assuntos referentes à saúde bucal. Os casos de urgência foram encaminhados para o projeto de extensão Acolhendo Sorrisos Especiais da FO-UFPel.

Os dados coletados, no período de abril a maio de 2014, foram digitados duas vezes por duas pessoas, de forma independente, no Excel e validados no software EpiData 3.1. A análise foi realizada no programa Stata versão 10.0. Foram realizadas análises estatísticas descritivas, por meio de valores absolutos e relativos, e para a avaliação da associação entre as variáveis independentes e o desfecho do estudo foram utilizados os testes Qui-Quadrado, Exato de Fisher e Mann-Whitney, com significância de $5 \%$.

\section{Resulltados}

Dos 61 alunos matriculados na escola, 57 $(93,4 \%)$ foram selecionados para o estudo, segundo os critérios de inclusão, sendo que sete pais ou responsáveis não autorizaram a participação de seus filhos, totalizando, portanto, 50 escolares aptos para o estudo.

Neste estudo, a idade dos escolares variou entre 6 e 19 anos, com média de 12,2 anos ( $\mathrm{DP}=3,34)$. Quanto ao gênero, 21 (42\%) escolares pertenciam ao sexo feminino e 29 (58\%), ao masculino. A distribuição dos escolares com deficiência auditiva segundo experiência de cárie e características demográficas, grau de deficiência auditiva e consulta ao cirurgião-dentista está apresentada na Tabela 1. Não foi observada diferença estatisticamente significativa entre experiência de cárie e as variáveis estudadas $(\mathrm{p}>0,05)$. 
Tabela 1 - Distribuição dos escolares com deficiência auditiva segundo experiência de cárie e características demográficas, grau de deficiência auditiva e consulta ao cirurgião-dentista, Pelotas, $2016(n=50)$

\begin{tabular}{|c|c|c|c|c|c|c|c|}
\hline \multirow{3}{*}{ Variáveis } & \multicolumn{4}{|c|}{ Experiência de cárie } & \multirow{2}{*}{\multicolumn{2}{|c|}{ Total }} & \multirow{3}{*}{$\mathrm{p}$} \\
\hline & \multicolumn{2}{|c|}{ Presente } & \multicolumn{2}{|c|}{ Ausente } & & & \\
\hline & $\mathrm{N}$ & $\%$ & $n$ & $\%$ & $\mathrm{n}$ & $\%$ & \\
\hline \multicolumn{8}{|l|}{ Sexo } \\
\hline Feminino & 11 & 52,4 & 10 & 47,6 & 21 & 100 & \multirow[t]{2}{*}{$0,8^{*}$} \\
\hline Masculino & 16 & 55,2 & 13 & 44,8 & 29 & 100 & \\
\hline \multicolumn{8}{|l|}{ Idade } \\
\hline 6 a 12 anos & 13 & 54,2 & 11 & 45,8 & 24 & 100 & \multirow[t]{2}{*}{$0,9^{*}$} \\
\hline 13 a 19 anos & 14 & 53,8 & 12 & 46,2 & 26 & 100 & \\
\hline \multicolumn{8}{|c|}{ Grau de deficiência auditiva } \\
\hline Ouve parcialmente & 14 & 56,0 & 11 & 44,0 & 25 & 100 & \multirow[t]{2}{*}{$0,7^{*}$} \\
\hline Totalmente surdo & 13 & 52,0 & 12 & 48,0 & 25 & 100 & \\
\hline
\end{tabular}

Consulta ao cirurgião-dentista

\begin{tabular}{l|c|c|c|c|c|c|c}
\hline Sim & 26 & 56,5 & 20 & 43,5 & 46 & 100 & $0,2^{* *}$ \\
Não & 1 & 25,0 & 3 & 75,0 & 4 & 100 & \\
\hline
\end{tabular}

Fonte: dados da pesquisa.

Em relação ao grau de deficiência auditiva, 25 escolares ouviam parcialmente $(50 \%)$ e $25(50 \%)$ eram totalmente surdos. Dentro desses grupos, verificou-se que $15(30 \%)$ deles apresentavam algum tipo de deficiência associada: hiperatividade $(\mathrm{n}=2)$, esquizofrenia $(n=1)$, deficiência visual $(n=2)$, problemas neurológicos $(n=5)$, paralisia cerebral leve $(\mathrm{n}=2)$ e deficiência intelectual $(\mathrm{n}=3)$.

A distribuição dos escolares de acordo com presença de deficiências associadas segundo componentes do índice CPO-D, média do CPO-D, presença de placa e sangramento gengival na sondagem está apresentada na Tabela 2 . Não foi observada associação estatisticamente significativa entre a presença de deficiência associada e as variáveis estudadas ( $\mathrm{p}$ $>0,05$ ).

Tabela 2 - Distribuição dos escolares de acordo com presença de deficiências associadas segundo experiência de cárie, componentes do índice CPO-D, média do CPOD, presenca de placa sangramento gengival na sondagem, Pelotas, $2016(n=49)$

\begin{tabular}{|c|c|c|c|c|c|c|c|}
\hline \multirow{3}{*}{ Variáveis } & \multicolumn{4}{|c|}{ Deficiência associada* } & \multirow{2}{*}{\multicolumn{2}{|c|}{ Total }} & \\
\hline & \multicolumn{2}{|c|}{$\begin{array}{c}\text { Presente } \\
15(30,6 \%)\end{array}$} & \multicolumn{2}{|c|}{$\begin{array}{c}\text { Ausente } \\
34(69,4 \%)\end{array}$} & & & \\
\hline & $\mathrm{N}$ & $(\%)$ & $\mathrm{N}$ & $(\%)$ & $n$ & $(\%)$ & $\mathrm{P}$ \\
\hline \multicolumn{8}{|c|}{ Experiência de cárie } \\
\hline Presente & $11(42,3)$ & & $15(57,7)$ & & $26(100)$ & & 0,06 \\
\hline Ausente & $4(17,4)$ & & $19(82,6)$ & & $23(100)$ & & \\
\hline \multicolumn{8}{|c|}{ Componentes do CPO-D } \\
\hline Cariado (C) & $10(41,7)$ & & $14(58,3)$ & & $24(100)$ & & 0,10 \\
\hline Perdido (P) & $3(42,9)$ & & $4(57,1)$ & & $7(100)$ & & 0,45 \\
\hline Obturado (O) & $4(40,0)$ & & $6(60,0)$ & & $10(100)$ & & 0,47 \\
\hline Média do CPO-D & 2,53 & & 1,59 & & 1,88 & & $0,11^{* *}$ \\
\hline \multicolumn{8}{|l|}{ Placa supragengival } \\
\hline Presente & $12(36,7)$ & & $32(71,1)$ & & $44(100)$ & & 0,29 \\
\hline Ausente & $3(60,0)$ & & $2(40,0)$ & & $5(100)$ & & \\
\hline \multicolumn{8}{|c|}{ Sangramento gengival } \\
\hline Presente & $2(18,2)$ & & $9(81,8)$ & & $11(100)$ & & 0,51 \\
\hline Ausente & $13(34,2)$ & & $25(65,8)$ & & $38(100)$ & & \\
\hline
\end{tabular}

* Perda de informação; ** Teste Mann-Whitney.

Fonte: dados da pesquisa.
A experiência de cárie dentária foi de 54\% (CPO$-\mathrm{D}>0$ ), e a prevalência de cárie foi de $50 \%$. A média do índice CPO-D na população do estudo foi de 1,88 , e as médias nas faixas etárias de 6 a 12 e de 13 a 19 anos foram de 2,33 e 1,46, respectivamente.

Das famílias avaliadas, 32 (64\%) possuíam renda média de um salário mínimo (R\$ 788,00), 7 (14\%) apresentaram renda até $\mathrm{R} \$ 500,00$ e 10 famílias (20\%) relataram ter renda de mais de $\mathrm{R} \$ 800,00$. Em um dos questionários, a pergunta sobre a renda não foi respondida (2\%).

Em relação à escolaridade dos pais/cuidadores, $29(58 \%)$ não completaram o ensino fundamental, 12 (24\%) estudaram de 9 a 11 anos e $9(18 \%)$ relataram ter 12 anos ou mais de estudo. Não houve associação estatisticamente significativa entre experiência de cárie e renda $(p=0,2)$ e escolaridade $(p=0,4)$.

Em relação à ocupação dos pais/cuidadores, 35 (70\%) eram do lar, 7 (14\%) relataram trabalhar fora em apenas um turno, $6(12 \%)$ responderam que trabalham fora mais de um turno e 2 responsáveis não responderam a essa questão (4\%).

Quando perguntados sobre quem realiza a escovação, 31 responsáveis $(62 \%)$ relataram que é o próprio escolar, $4(8 \%)$ relataram que é o cuidador e 15 $(30 \%)$ que ambos a realizam. Os recursos mais citados para a realização da higiene bucal foram escova e dentifrício fluoretado para 31 escolares (62\%), 13 relataram utilizar também fio dental (26\%) e 6 escolares $(12 \%)$ afirmaram usar, ainda, enxaguante bucal.

\section{Discussão}

A experiência de cárie dos escolares examinados neste estudo foi semelhante aos resultados encontrados por outros autores que também avaliaram indivíduos com deficiência auditiva ${ }^{4-6,9} \mathrm{e}$ aos dados da população brasileira sem deficiência (SB Brasil, 2010) ${ }^{15}$.

No entanto, no grupo de escolares com outras deficiências associadas, o índice CPO-D foi maior $(2,53)$, quando comparado ao grupo com apenas deficiência auditiva (1,59). A deficiência auditiva, diferente de outros tipos de deficiência que comprometem coordenação motora e autonomia, não parece ser limitante para a higiene bucal.

Neste estudo, houve uma distribuição uniforme em relação à idade e uma maior prevalência de deficiência auditiva no sexo masculino, corroborando com outros autores ${ }^{6,7,9,10,12}$. Em relação à escolaridade e à renda dos pais/responsáveis, não foi observada associação com experiência de cárie, embora a literatura em geral aponte associação entre o baixo nível socioeconômico e a ocorrência de cárie dentária $^{8}$. A maioria dos cuidadores classificou sua ocupação como do lar (70\%), o que talvez explique a baixa renda familiar, assim como evidenciado no estudo de Martinez $^{9}$ (2000). O fato de o cuidador não ter uma atividade fora de casa talvez se justifique pelos cuidados e pela atenção que o Paciente com Necessi- 
dades Especiais (PNE) necessita, embora a maioria dos pais ou cuidadores tenha relatado não auxiliar o escolar durante a escovação. Dessa forma, ações de educação em saúde bucal com os cuidadores podem potencializar os cuidados com os PcDs.

Embora esses indivíduos tenham uma higiene bucal insatisfatória, apenas 11 alunos apresentaram sangramento gengival na sondagem. O número expressivo de escolares que fazem uso do fio dental rotineiramente (38\%) também pode justificar o baixo ISG. Além disso, IPV elevado e ISG baixo sugerem que a placa bacteriana depositada sobre os dentes é recente e que o escolar consegue, normalmente, manter uma condição bucal satisfatória.

Não foi observada diferença nos índices que avaliam higiene bucal entre os grupos com e sem deficiência associada, talvez porque o grau de deficiência associada era leve. Crianças com deficiência neuropsicomotora, principalmente aquelas com maior comprometimento motor, tendem a ter pior condição de higiene bucal ${ }^{16}$.

A maioria dos trabalhos que envolvem PcD apresenta uma amostra reduzida, pela dificuldade de acesso aos registros desses pacientes e pela variabilidade metodológica que dificulta a comparação entre os estudos ${ }^{4,5,9,10,17}$. Embora crianças com deficiência auditiva leve estejam matriculadas na rede regular de ensino, este estudo avaliou crianças e jovens em uma escola de educação especial do município de Pelotas, dessa forma, os achados podem refletir a situação de saúde bucal da parcela mais comprometida.

Assim, estratégias educativas e preventivas que possibilitem um controle efetivo da placa, direcionadas a crianças e jovens com deficiência auditiva, devem ser estimuladas, a fim de proporcionar melhores condições de saúde bucal e despertar o autocuidado. Sugere-se que a língua de sinais seja uma ferramenta utilizada pelo profissional de saúde, pois facilita a interação e a comunicação com esses indivíduos.

\section{Conclusões}

A condição de higiene bucal dos escolares com deficiência auditiva avaliados foi insatisfatória. A doença cárie esteve presente em uma parcela considerável. Dessa forma, estratégias de saúde bucal devem ser direcionadas a escolares, assim como aos seus cuidadores, visando à possibilidade de os indivíduos com deficiência auditiva serem estimulados para o autocuidado em saúde.

\section{Agradecimentos}

Os autores agradecem à direção da Escola Especial Professor Alfredo Dub por permitir a realização do estudo e às crianças e aos seus familiares pela participação.

\section{Abstract}

Objective: To assess the oral health status of schoolchildren aged 6 to 19 years with hearing impairment at a special education school in Southern Brazil. Methods: This prospective cross-sectional study collected data on socioeconomic status, degree of hearing loss, and oral hygiene habits, through a questionnaire directed at caregivers. Oral health status was obtained by a clinical exam, which assessed the Visible Plaque Index, the Gingival Bleeding Index, and the Decayed, Missing, Filled Teeth Index (dmft and DMFT). Descriptive statistical analysis was performed with absolute and relative values, using the Stata software version 10.0. The association between independent variables and the study outcome was assessed with chi-square, Fisher's Exact, and Mann-Whitney tests, at 5\% significance level. Results: Fifty students were assessed, whereas the majority was male, low income, and the guardian was a housewife. Regarding oral health, $90 \%$ of the students had visible plaque, $22 \%$ had gingival bleeding on probing, and the mean $\mathrm{dmft} / \mathrm{DMFT}$ index was 1.8. Conclusions: The oral hygiene status of the assessed students with hearing impairment was unsatisfactory, and caries disease was present in a significant portion of the children, but it was similar to that of the population with no disabilities.

Keywords: Dental caries. Students. Persons with hearing impairments. Oral health.

\section{Referências}

1. World Health Organization [homepage]. Geneva: World Health Organization; 1997. [citado 2016 jun. 23]. Disponível em URL: http://www.who.int/en/ORGANIZATIONWH.

2. Haddah AS, Guaré RO, Ortega AOL. Pacientes com necessidades especiais. 8. ed. São Paulo: Santos; 2012. p. 967-1003.

3. Campos CC, Frazão BB, Saddi GL, Morais LA, Ferreira MG, Setúbal PCO, et al. Manual prático para o atendimento odontológico de pacientes com necessidades especiais. 2. ed. Goiânia; 2009. p. 104.

4. Giro EMA, Orrico SRP, Campos JADB, Lorena SM, Cortez LMS. Prevalência de cárie em pacientes com necessidades especiais institucionalizados ou não-institucionalizados: consumo de carboidratos simples. Rev Odontol Unesp 2004; 33(2):75-9.

5. Jain M, Mathur A, Kumar S, Dagli RJ, Duraiswamy P, Kulkarni S. Dentition status and treatment needs among children with impaired hearing attending a special school for the deaf and mute in Udaipur, India. J Oral Sci 2008; 50(2):161-5.

6. Gondin LAM, Andrade MC, Maciel SSSV, Ferreira MAF. Perfil epidemiológico das condições dentárias e necessidade de tratamento dos portadores de deficiência da cidade de Caruaru, Pernambuco, Brasil. Rev Gauch Odontol 2008; 56(4):393-7.

7. Möller CDC, Ibaldo LTS, Tovo MF. Avaliação das condições de saúde bucal de escolares deficientes auditivos no município de Porto Alegre, RS, Brasil. Pesq Br Odontoped Clin Integr 2010; 10(2):195-200.

8. Lucca MQ, Loureiro CA. Experiência de cárie em indivíduos com paralisia cerebral. Rev Gauch Odontol 2011; 59(3):387-95. 
9. Martinez NL. Experiencia de caries dental en niños con deficiencias auditivas en dos escuelas del área metropolitana de Caracas, Venezuela. Acta Odontol Venez 2000; 41:4-8.

10. Souza Filho MD, Nogueira SDM, Martins MCC. Avaliação da saúde bucal de deficientes visuais em Teresina-PI. Arq Odontol 2010; 45(2):66-74.

11. Dall'Magro AK, Dall'Magro E, Kuhn GF. Perfil clínico dos pacientes especiais tratados sob anestesia geral no Hospital São Vicente de Paulo de Passo Fundo entre os anos de 2005 e 2010. RFO UPF 2010; 15(3):253-6.

12. Romanelli MCMOV. Levantamento epidemiológico das doenças cárie e periodontal em alunos com necessidades especiais das APAEs na região metropolitana de Curitiba - Paraná [Dissertação de Mestrado]. Ponta Grossa: Universidade Estadual de Ponta Grossa; 2006.

13. Ainamo J. Epidemiologia da doença periodontal. In: Koogan G. Tratado de Periodontologia Clínica. Rio de Janeiro; 1992. p. $42-57$.

14. Greene JC, Vermillion JR. The Simplified Oral Hygiene Index. J Am Dent Assoc 1964; 68:7-13.

15. Ministério da Saúde. Secretaria de Atenção à Saúde. Departamento de Atenção Básica [homepage]. Porto Alegre: Projeto SB RS: condições de saúde bucal da população do Rio Grande do Sul. Secretaria da Saúde do Estado do Rio Grande do Sul, Divisão de Atenção à Saúde, Seção de Saúde Bucal; 2003. [citado 2003 jul.]. Disponível em URL: http://www1. saude.rs.gov.br/dados/1165251847559e\%20Relat\%F3rio\%20 T\%E9cnico\%20da\%20Macrorregi\%E3o\%20Norte.pdf.

16. Nasiloski KS, Silveira ER, César Neto JB, Schardosim LR. Evaluation of periodontal conditions and oral hygiene in school children with neuropsychomotor disorders. Rev Odontol Unesp 2015; 44(2):103-7.

17. Costa FS, Neves LB, Bonow MLM, Azevedo MS, Schardosim LR. Efetividade de uma estratégia educacional em saúde bucal aplicada a crianças deficientes visuais. RFO UPF 2012; 17(1):12-7.

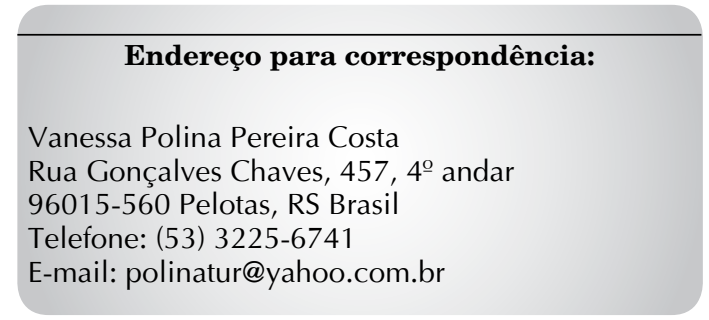

Recebido: 22/08/2016. Aceito: 15/09/2016. 